\title{
Effect of gender and clinical-financial vulnerability on mental distress due to COVID-19
}

\author{
Aditya Simha $^{1}$ (D) $\cdot$ Ramakrishna Prasad $^{2} \cdot$ Sana Ahmed $^{3} \cdot$ Naren P. Rao $^{4}$ \\ Received: 28 August 2020 / Accepted: 2 December 2020 / Published online: 9 January 2021 \\ (C) The Author(s), under exclusive licence to Springer-Verlag GmbH, AT part of Springer Nature 2021
}

\begin{abstract}
The COVID-19 pandemic has had an enormous impact on people's mental health. This study examines whether gender influences the mental distress in people from at-risk populations (clinically high risk and financially vulnerable). A crosssectional survey was administered between the 23rd and 28th of April 2020 to 15,691 individuals from 32,596 households in the UK. Our findings confirmed that individuals who are clinically high risk or financially vulnerable or a combination of both experience significantly higher levels of mental distress. Additionally, we also found that females experienced higher levels of mental distress than males across various categories.
\end{abstract}

Keywords COVID-19 $\cdot$ Mental health $\cdot$ Mental distress $\cdot$ Gender

\section{Introduction}

This COVID-19 pandemic has considerably increased global mental health issues (Druss 2020; Holmes et al. 2020; Li et al. 2020, b; Nanjundaswamy et al. 2020; Yao et al. 2020). Overall mental distress is further exacerbated by the emphasis on social distancing, and the shutdown of usual day-to-day functioning. For instance, the prevalence of mental distress was $27.3 \%$ in April 2020 compared to $18.9 \%$ in 2018-2019 (Pierce et al. 2020; Galea et al. 2020). While COVID-19 affects the entire population, fatal outcomes are seen in certain subgroups like the elderly and those with existing medical conditions.

Additionally, many individuals are now having to deal with precarious financial conditions, due to reduced hours at work,

Women's mental health during the Covid-19 pandemic.

Edited by: Anita Riecher-Rössler

Aditya Simha

simhaa@uww.edu

1 University of Wisconsin, Whitewater, WI, USA

2 PCMH Restore Health, National Center for Primary Care Research \& Policy, Academy of Family Physicians of India (AFPI), Bengaluru, India

3 Chicago, IL, USA

4 National Institute of Mental Health and Neurosciences, Bengaluru, India or being laid off, or due to their employers not faring well in this new climate. Put together, individuals who are at clinically high risk and financially vulnerable are likely to experience elevated mental distress compared to the general population. However, the modulating effect of gender on subjective mental distress is not well examined. Therefore, we examined whether the subjective mental distress in these at-risk populations (clinically high risk and financially vulnerable) is influenced by the gender of an individual.

\section{Measures}

We used a publicly available database by the Institute for Social and Economic Research, UK, between April 23, 2020, and April 28, 2020. The data was collected by the Institute for Social and Economic Research, after obtaining institutional approval from the University of Essex. We did not need to obtain additional approval in order to analyze or use the data. The Understanding Society COVID-19 survey (Institute for Social and Economic Research 2020) sampling frame included participants from probability samples of postal addresses throughout the UK. The survey was administered to 32,596 individuals, and the final usable sample of 15,691 individuals indicated a $48.13 \%$ response rate.

After data cleaning, the sample used for analysis comprised 15,691 individuals (58.4\% females). The mental distress variable was derived from the responses to the 12-item general 
Table 1 Intercorrelations

\begin{tabular}{|c|c|c|c|c|c|c|c|}
\hline & Variable & Mean & $\mathrm{SD}$ & 1 & 2 & 3 & 4 \\
\hline 1 & Age & 51.48 & 16.75 & 1 & & & \\
\hline 2 & Gender & 1.58 & 0.49 & $-0.09 * *$ & 1 & & \\
\hline 3 & Clinical vulnerability & 0.31 & 0.46 & $0.517 * *$ & $-0.07 * *$ & 1 & \\
\hline 4 & Financial vulnerability & 1.92 & 0.89 & $-0.15^{* *}$ & $0.028 * *$ & $-0.06^{* *}$ & 1 \\
\hline 5 & Mental distress & 12.48 & 6.03 & $-0.18^{* *}$ & $0.171 * *$ & $-0.05^{* *}$ & $0.333 * *$ \\
\hline
\end{tabular}

health questionnaire (GHQ-12). GHQ-12 has a range of 1-36 with higher scores indicating greater mental distress. Additionally, we obtained data on financial vulnerability through a question on the survey that assessed how financially vulnerable the respondents thought they were. These responses were categorized as very low levels of vulnerability, low levels of vulnerability, medium levels of vulnerability, high levels of vulnerability, and extreme levels of vulnerability. This measure was anchored and correlated with other measures such as income and employment.

Similarly, we obtained data on whether respondents were clinically at high risk from COVID-19 through a single-item question that asked respondents if they were clinically at high risk. This measure was anchored and correlated with other measures such as age and comorbidities. The data was obtained from the UK Data Service on June 9, 2020, and the intercorrelation and multivariate regression analysis was conducted using SPSS version 26. Our main dependent variable was mental distress as assessed by scores on the GHQ-12, while our independent variables were financial vulnerability and clinical vulnerability and gender.

We first did a univariate analysis to examine the data and, only after that, did our multivariate analyses. Our univariate analyses revealed that none of the variables in our univariate (i.e., those that were entered in the multivariate model) explained a large amount of variance, as when compared to the multivariate model.

\section{Results}

The average age of the respondents was 51.2 years. $31.2 \%$ of the respondents were clinically high risk and $21 \%$ were financially vulnerable. The average mental distress score was 12.48 indicating moderate levels of mental distress. The results from the multivariate regression analysis revealed that individuals who were clinically at high risk from COVID-19 were more likely to experience mental distress $(\beta=0.026$, $95 \%$ CI, LLCI $=0.862, \mathrm{ULCI}=1.617$ ).

Similarly, the results indicated that individuals who perceived that they were financially vulnerable due to COVID19 were more likely to experience mental distress $(\beta=0.294$, $95 \% \mathrm{CI}, \mathrm{LLCI}=1.88, \mathrm{ULCI}=2.09$ ). There was a significant gender-specific effect on the subjective distress reported, with females reporting greater levels of mental distress as compared to males $(\beta=0.152,95 \% \mathrm{CI}, \mathrm{LLCI}=1.680, \mathrm{ULCI}=$ $2.035)$. Table 1 and 2 present the results of the intercorrelations and regression results.

\section{Discussion}

Our findings confirmed our hypothesis that individuals who are clinically at high risk or financially vulnerable or a combination of both experience significantly higher levels of mental distress. A novel and important finding is the significant influence of gender; females experienced significantly greater distress as compared to males. This was also true when it came to interactions of gender and financial vulnerability. Across levels of financial vulnerability, females had significantly higher levels of mental stress than males. Similarly, females who were in the high clinical risk category had higher levels of mental distress than the males who were in the high clinical risk category.

Several factors are possible reasons for increased vulnerability of women during this pandemic such as lack of financial independency, occupational exhaustion, work-family conflict,

Table 2 Regression results

\begin{tabular}{lccccc}
\hline Variable & B (unstd) & $\beta$ (std Beta) & LLCI & ULCI & $\Delta$ R2 \\
\hline Age & $-0.052 * * *$ & $-0.143 * * *$ & -0.057 & -0.046 & \\
Gender & $1.86^{* * *}$ & $0.152^{* * *}$ & 1.68 & 2.04 \\
Financial vulnerability & $1.98^{* * *}$ & $0.294 * * *$ & 1.88 & 2.09 \\
Clinical vulnerability & $1.24 * * *$ & $0.026 * * *$ & 0.862 & 1.617 & \\
& & & & & 0.155 \\
$* * * p<0.001$ & & & &
\end{tabular}


and inequality in domestic labor ( $\mathrm{Li}$ and Graham 2017). In addition, infection-reducing measures such as lockdowns are associated with heightened domestic violence (Gulati and Kelly 2020). These socioeconomic factors together with the biological vulnerability due to sex hormones ( $\mathrm{Li}$ and Graham 2017) could augment the vulnerability to mental distress among women. In addition, emerging evidence also suggests fear of perinatal COVID infection, and feeling unprepared for birth due to the pandemic adds to the stress in pregnant women (Preis et al. 2020).

These findings need to be interpreted in the context of a few limitations. Considering the extraordinary circumstances under which the research was conducted, a self-report questionnaire was used, and no follow-up interviews were conducted. Hence, disadvantages associated with self-report studies such as non-response bias, social desirability bias, recall bias, and missing data are possible. Additionally, this research only reports one wave of the COVID-19 study, future waves, and further longitudinal analyses may reveal other findings.

These findings could certainly have important implications, worldwide and not just in the UK. Considering a high prevalence of higher distress in vulnerable groups, public health interventions to reduce mental distress need to prioritize targeting these subgroups. Among the high-risk groups, women need special attention and the interventions need to be sensitive to address gender-specific issues. In addition to the medical care, allaying the financial difficulties might also decrease the distress. Finally, systematic studies are needed to examine the underlying reasons for this greater mental distress among women. Additionally, we also encourage future studies to examine the impact of COVID-19 on individuals from the LGBTQ communities, as we do believe that the COVID19 pandemic would have inordinately deleterious effects for LGBTQ individuals.

\section{Compliance with ethical standards}

Conflict of interest The authors declare that they have no conflict of interest.

\section{References}

Druss BG (2020) Addressing the COVID-19 pandemic in populations with serious mental illness. JAMA Psychiatry

Galea S, Merchant RM, Lurie N (2020) The mental health consequences of COVID-19 and physical distancing: the need for prevention and early intervention. JAMA Intern Med

Gulati G, Kelly BD (2020) Domestic violence against women and the COVID-19 pandemic: what is the role of psychiatry? Int J Law Psychiatry 71:101594

Holmes EA, O'Connor RC, Perry VH, Tracey I, Wessely S, Arseneault L, Ballard C, Christensen H, Silver RC, Everall I, Ford T (2020) Multidisciplinary research priorities for the COVID-19 pandemic: a call for action for mental health science. Lancet Psychiatry

Li SH, Graham BM (2017) Why are women so vulnerable to anxiety, trauma-related and stress-related disorders? The potential role of sex hormones. Lancet Psychiatry 4(1):73-82

Li G, Miao J, Wang H, Xu S, Sun W, Fan Y, Zhang C, Zhu S, Zhu Z, Wang W (2020) Psychological impact on women health workers involved in COVID-19 outbreak in Wuhan: a cross-sectional study. J Neurol Neurosurg Psychiatry 91(8):895-897

Li J, Yang Z, Qiu H, Wang Y, Jian L, Ji J, Li K (2020) Anxiety and depression among general population in China at the peak of the COVID-19 epidemic. World Psychiatry 19(2):249-250

Nanjundaswamy MH, Shiva L, Desai G, Ganjekar S, Kishore T, Ram U, Satyanarayana V, Thippeswamy H, Chandra PS (2020) COVID-19related anxiety and concerns expressed by pregnant and postpartum women - a survey among obstetricians. Arch Womens Ment Health. https://doi.org/10.1007/s00737-020-01060-w

Pierce M, Hope H, Ford T, Hatch S, Hotopf M, John A, Kontopantelis E, Webb R, Wessely S, McManus S, Abel KM (2020) Mental health before and during the COVID-19 pandemic: a longitudinal probability sample survey of the UK population. Lancet Psychiatry 7(10): 883-892

Preis H, Mahaffey B, Heiselman C, Lobel M (2020) Vulnerability and resilience to pandemic-related stress among U.S. women pregnant at the start of the COVID-19 pandemic. Soc Sci Med 266:113348

University of Essex, Institute for Social and Economic Research (2020) Understanding society: COVID-19 study, 2020. [data collection]. UK Data Service. SN: 8644 https://doi.org/10.5255/UKDA-SN8644-1

Yao H, Chen JH, Xu YF (2020) Patients with mental health disorders in the COVID-19 epidemic. Lancet Psychiatry 7(4):e21

Publisher's note Springer Nature remains neutral with regard to jurisdictional claims in published maps and institutional affiliations. 